\title{
Klinisch-chemische Untersuchungen bei zwei Patienten nach homologer Lebertransplantation
}

\author{
Von J. Breuer, T. S. Lie und H. Breuer \\ Aus der Cbirurgischen Universitätsklinik und Poliklinik Bonn und dem Institut fïr Klinische Bioclsemie und Klinische Cbemie \\ der Universität Bonn
}

(Eingegangen am 3. Februar 1970)

Bei 2 mānnlichen Patienten (Alter 30 und 50 Jahre) mit cinem Neoplasma der Leber wurden nach Transplantation einer menschlichen Leber während der gesamten postoperativen Phase folgende klinisch-chemischen Untersuchungen im Serum durchgeführt: Bilirubin, Alanin-Aminotransferase, Aspartat-Aminotransferase, alkalische Phosphatase, Lactat-Dehydrogenase, Glutamat-Dehydrogenase und Cholinesterase. Die Verlaufsbeobachtungen erstreckten sich bei dem 30 jährigen Patienten über 206 Tage und bei dem 50 jährigen Patienten über 6 Tage. Die Ergebnisse der klinisch-chemischen Untersuchungen konnten mit dem klinischen Verlauf nach der homologen Lebertransplantation weitgehend korreliert werden. Neben der Alanin- und Aspartat-Aminotransferase und alkalischen Phosphatase hat sich das Verhalten der Glutamat-Dehydrogenase und der Cholinesterase als besonders aufschlußreich bei der Beurteilung der Funktion der transplantierten Leber erwiesen.

\section{Biochemical investigations in 2 patients after homotransplantation of the liver}

After homotransplantation of the liver in two male patients (aged 30 and 50 years) with primary hepatic malignoma, the following biochemical determinations in serum were carried out during the postoperative phase: bilirubin, alanine aminotransferase, aspartate aminotransferase, alkaline phosphatase, lactate dehydrogenase, glutamate dehydrogenase and cholinesterase. The investigations were performed during a period of 206 days in the 30 year old patient and during a period of 6 days in the 50 year old patient. The results of the biochemical investigations correlated fairly well with the postoperative course after transplantation of the liver. In addition to transaminases and alkaline phosphatase, the measurement of the activities of glutamate dehydrogenase and cholinesterase have proved to be of particular value in evaluating the function of the transplanted liver.

In der vorliegenden Arbeit wird über klinisch-chemische Untersuchungen bei zwei männlichen Patienten nach Transplantation einer menschlichen Leber berichtet. Bei dem ersten Patienten handelte es sich um einen 30 jährigen Mann (V. G.) mit einem Neoplasma der Leber; die transplantierte Leber wurde einem 31jährigen Organspender entnommen, nachdem er an einer Gehirnblutung verstorben war (1). Ein Teil der in der unmittelbaren postoperativen Phase (21 Tage post op.) bei dem Patienten V. G. erhobenen Befunde sind bereits früher mitgeteilt worden $(1,2)$. Der zweite, 50 jährige Patient (H. O.), litt ebenfalls an einem Neoplasma der Leber sowie an einer histologisch gesicherten Lebercirrhose; die transplantierte Leber stammte von einem 17 jährigen Organspender, der an einem Hirnaneurysma gestorben war. Die im folgenden beschriebenen Ergebnisse, die einen Einblick in die Funktion der transplantierten Organe ermöglichen sollen, erstrecken sich bei dem ersten Patienten (V. G.) über einen Zeitraum von 206 Tageñ und bei dem zweiten Patienten (H. O.) über einen Zeitraum von 6 Tagen.

\section{Methodik}

\section{Organentnabme und Empfängeroperation}

Angaben über die technischen Einzelheiten der Organentnahme und der Einpflanzung des Spenderorgans bei dem Patienten V. G. finden sich bei (1). Einzelheiten über das operative Vorgehen bei dem zweiten Patienten ( $H$. O.) werden an anderer Stelle mitgeteilt (GürǵemanN und Mitarbeiter).

\section{Bestiminung der Enzymaletivitäten}

Die Aktivität der alkalischen Phosphatase (EC 3.1.3.1) wurde unter Verwendung von Natrium-p-phenylphosphat, die Aktivität der Cholinestrase (EC 3.1.1.8) unter Verwendung von Acetylthiocholin-jodid kolorimetrisch bestimmt (Biochemica-Test-Combination, C. F. Boehringer, Mannheim). Die Aktivitätsbestimmungen der Aspartat-Aminotransferase (EC 2.6.1.1), der AlaninAminotransferase (EC 2.6.1.2), der Lactat-Dehydrogenase (EC 1.1.1.27) und der Glutamat-Dehydrogenase (EC 1.4.1.3) erfolgten im kinetischen UV-Test bei $366 \mathrm{~nm}$ (Biochemica-TestCombination, C. F. Boshringer, Mannheim).

\section{Sonstige Bestimmungen}

Gesamtbilirubin und konjugiertes Bilirubin wurden mit Sulfanilsäure (Biochemica-Test-Combination, C. F. Boehringer, Mannheim) bestimmt, Gesamteiweiß mit der Biuret-Reaktion, Kreatinin mit Pikrinsäure in alkalischer Lösung (BiochemicaTest-Combination, C. F. Boehringer, Mannheim), Harnstoff enzymatisch mit Urease (Biochemica-Test-Combination, C. F. Boehringer, Mannheim) und Natrium sowie Kalium flammenphotometrisch (Flammenphotometer Eppendorf).

\section{Ergebnisse und Diskussion}

Verlaufsbeobacbtungen bein Patienten V. G.

Das Verhalten des Gesamtbilirubins und der alkalischen Phosphatase ist in Abbildung 1 wiedergegeben, das der Transaminasen in Abbildung 2 und das der Lactatund Glutamat-Dehydrogenase und Cholinesterase in Abbildung 3. Die Verlaufsbeobachtung erstreckte sich über 206 Tage nach der Lebertransplantation. Bis zum 78. Tage post. op. befand sich der Patient in stationärer Behandlung; anschließend wurde er nach Hause entlassen und am 111. Tage post op. wieder in die Klinik aufgenommen. Nach Durchführung der Lebertransplantation wurde der Patient drei größeren operativen Eingriffen unterzogen. Am 36. Tage wurde ein Gallengangsverschluß beseitigt, am 48. Tage eine arterielle 


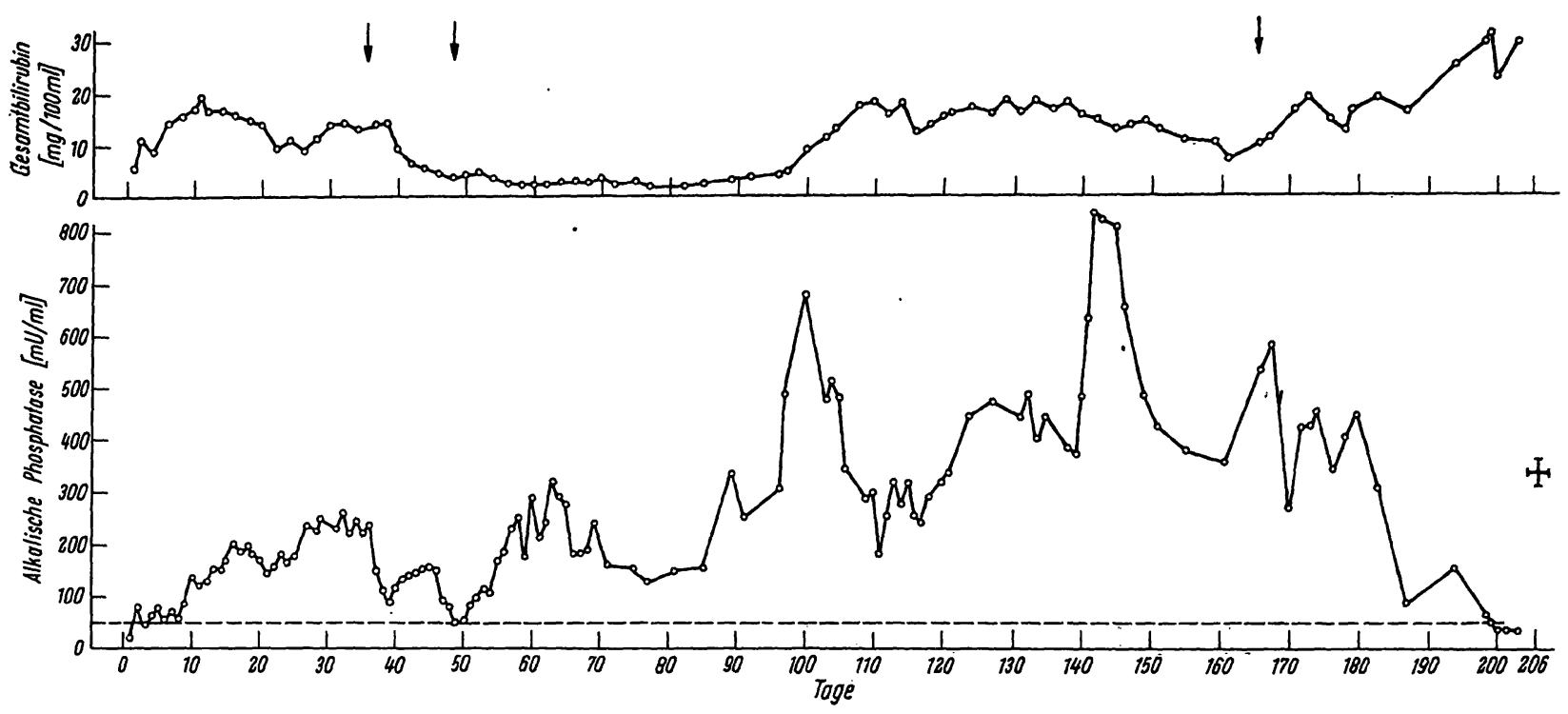

Abb. 1

Verhalten des Gesamtbilirubins (oben) und der alkalischen Phosphatase (unten) im Serum eines 30jährigen Patienten (V. G.) nach homologer Lebertransplantation. Die obere Begrenzung des Normalbereiches der alkalischen Phosphatase ist durch eine gestrichelte Linie gekennzeichnet. Am Tage 0 wurde die Transplantation vorgenommen. Die Pfeile bezeichnen die Tage, an denen die im Text erwähnten operativen Eingriffe durchgeführt wurden

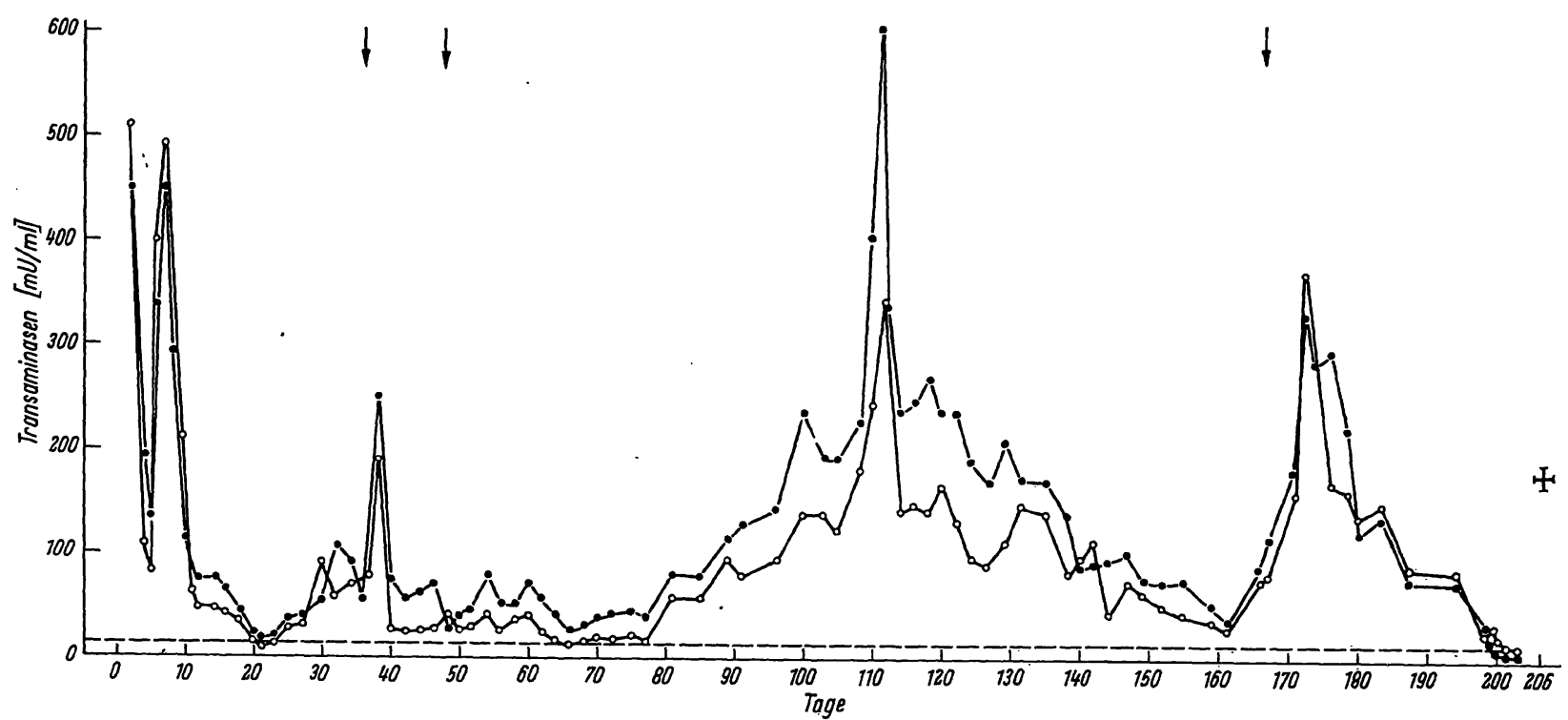

Abb. 2

Verhalten der Alanin-Aminotransferase (--0) und der Aspartat-Aminotransferase $(0-0)$ im Serum eines 30jährigen Patienten (V. G.) nach homologer Lebertransplantation. Die obere Grenze der Normalbereiche der Transaminasen sind durch gestrichelte Linien gekennzeichnet. Am Tage 0 wurde die Transplantation vorgenommen. Die Pfeile bezeichnen die Tage, an denen die im Text erwähnten operativen Eingriffe durchgeführt wurden

Blutung innerhalb des Transplantationsgebietes zum Stillstand gebracht und am 166. Tage eine Abscessincision in der Bauchhöhle durchgeführt.

Wie aus Abbildung 1 hervorgeht, stieg der Gesamtbilirubin-Gehalt des Blutes nach der Lebertransplantation zunächst auf Werte zwischen 10 und $20 \mathrm{mg} /$ $100 \mathrm{~m} /$ an. Nach etwa 40 Tagen erfolgte ein deutlicher Abfall; zwischen dem 60. und 90. Tag bewegte sich das Gesamtbilirubin zwischen 1 und $2 \mathrm{mg} / 100 \mathrm{ml}$, stieg dann aber wieder auf Werte von etwa $15 \mathrm{mg} / 100 \mathrm{ml}$ an und erreichte ante finem Höchstwerte von $30 \mathrm{mg} / 100 \mathrm{ml}$. Ein sehr unregelmäßiges Verhalten zeigte die Aktivität der alkalischen Phosphatase (Abb. 1), die während der ersten 30 Tage nach der Lebertransplantation stetig auf Werte bis zu etwa $250 \mathrm{mU} / \mathrm{ml}$ anstieg und nach Be- seitigung eines Gallengangsverschlusses wieder abnahm. Am 63., am 100. und am 142. Tage erreichte die alkalische Phosphatase erneut deutliche Maxima, von denen das dritte Maximum bei Werten über $800 \mathrm{mU} /$ $\mathrm{m} l$ lag.

In Abbildung 2 ist das Verhalten der Aktivitäten der Transaminase im Serum wiedergegeben. Die Aktivitäten der beiden Transaminasen verhalten sich während des gesamten Beobachtungszeitraums etwa gleichsinnig. Außer dem unmittelbaren postoperativen Maximum am 1. und 2. Tage wurden vier weitere Maxima beobachtet. Das erste Maximum trat am 7. Tage als Folge einer Abstoßungskrise auf, das zweite Maximum am 37. Tage nach Durchführung der Gallenoperation, der dritte - und für die Alanin-Aminotransferase zugleich 

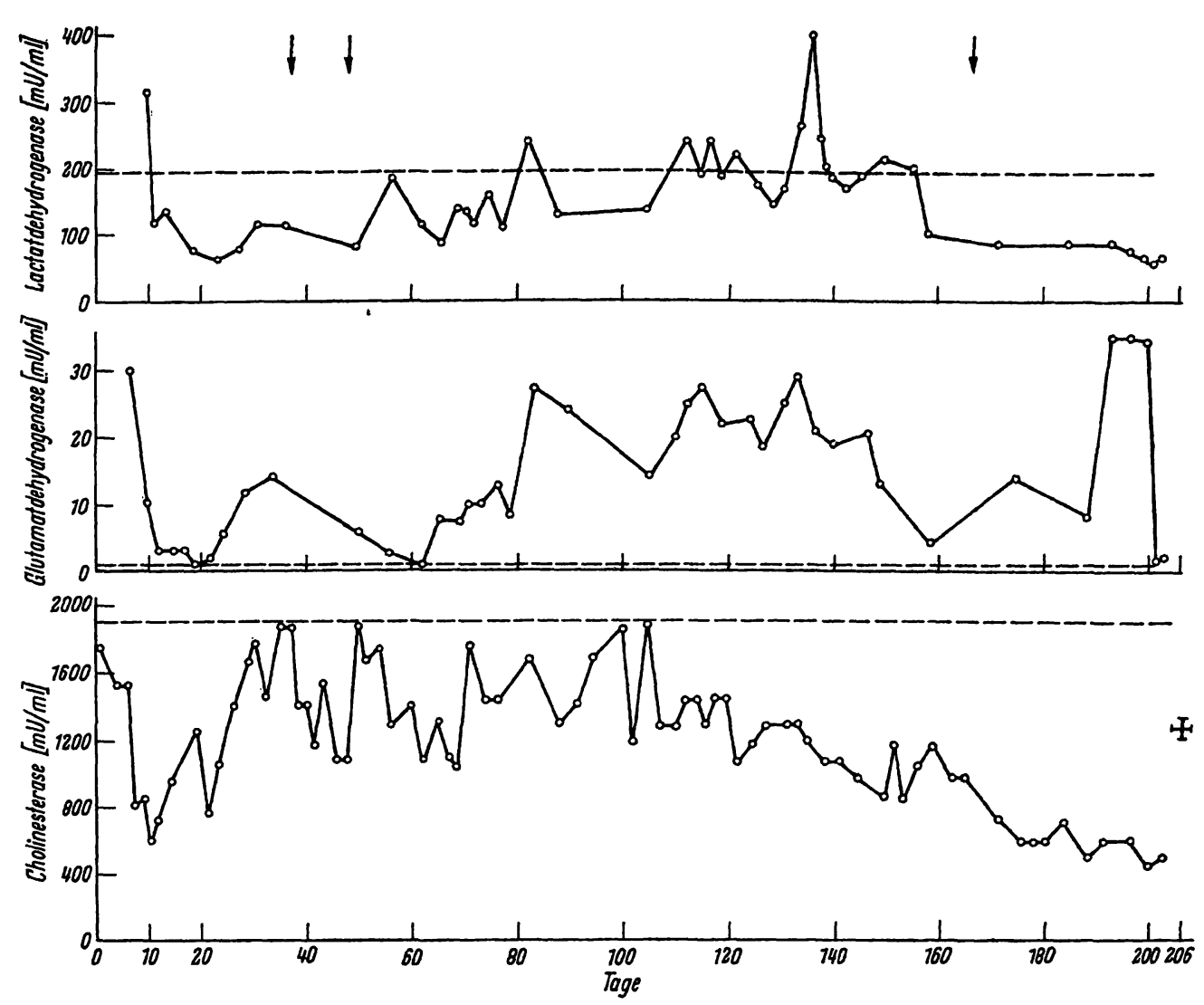

Abb. 3

Verhalten der Lactat-Dehydrogenase (oben), der Glutamat-Dehydrogenase (Mitte) und der Cholinesterase (unten) im Serum eines 30jährigen Patienten (V.G.) nach homologer Lebertransplantation. Für die Lactat- und die Glutamatdehydrogenase sind die oberen Grenzen, für die Cholinesterase ist die untere Grenze der Normalbereiche durch gestrichelte Linien gekennzeich-

I net. Am Tage 0 wurde die Transplantation vorgenommen. Die Pfeile bezeichnen die Tage, an denen die im Text erwähnten operativen Eingriffe durchgeführt wurden

höchste - Anstieg am 111. Tage und das vierte Maximum am 172. Tage. Die auffallende und stetige $\mathrm{Zu}-$ nahme der Aktivitäten der Transaminasen zwischen dem 78. und 111. Tage erfolgte während des Zeitraums, den der Patient in nicht-stationärer Behandlung zu Hause verbrachte; aufgrund des Anstiegs der Alanin-Aminotransferase auf $600 \mathrm{mU} / \mathrm{ml}$ erfolgte die Wiederaufnahme des Patienten.

Abbildung 3 zeigt das Verhalten der Aktivitäten der Lactat-Dehydrogenase, Glutamat-Dehydrogenase und Cholinesterase im Serum. Die Aktivität der LactatDehydrogenase bewegte sich, von wenigen Ausnahmen abgesehen, bis zum 150. Tage im oberen Bereich der Norm; nur am 135. Tage wurde ein Anstieg auf den doppelten Wert der obersten Normgrenze $(400 \mathrm{mU} / \mathrm{ml})$ beobachtet. Im Gegensatz zur LactatDehydrogenase waren die Aktivitäten der Glutamat-Dehydrogenase während des Beobachtungszeitraums im allgemeinen deutlich erhöht. Besonders hohe Werte wurden unmittelbar nach der Transplantation und wenige Tage ante finem erreicht. Nach einem anfänglichen Abfall der Cholinesterase mit einem Minimum nach 10 Tagen post op. stieg die Enzymaktivität wieder an und erreichte während der folgenden 100 Tage Werte bis zu $1900 \mathrm{mU} / \mathrm{ml}$ (untere Normgrenże). Vom 120. Tage an nahmen die Aktivitäten der Cholinesterase stetig bis auf Werte von etwa $400 \mathrm{mU} / \mathrm{m} / \mathrm{ab}$. Eine Interpretation der in Abbildung 1-3 dargestellten klinisch-chemischen Untersuchungen wird durch die Tatsache erschwert, daß der Patient V. G. im Anschluß an die Lebertransplantation neben Medikamenten und Infusionen zum Zwẹcke der Immunsuppression Cor- ticosteroide, Antimetaboliten und Antilymphocytenglobulin erhielt. Trotz dieser intensiven Behandlung und ihres Einflusses auf die Leberfunktion lassen sich einige allgemeine Rückschlüsse ziehen.

1. Die transplantierte Leber nahm unmittelbar nach der Einpflanzung ihre Funktion auf.

2. Die erste akute Abstoßungskrise dokumentierte sich in einem starken Anstieg der Transaminasen, in hohen Werten der Lactat-Dehydrogenase und der GlutamatDehydrogenase sowie in einem verzögerten, deutlichen Abfall der Cholinesterase.

3. Als Folge eines Gallengangsverschlusses wurde eine stetige Zunahmẹ der Aktivität der alkalischen Phosphatase beobachtet; nach Beseitigung des Verschlusses nahm die alkalische Phosphatase wieder ab, während die Transaminasen als Folge des operativen Eingriffs einen kurzdauernden Anstieg zeigten.

4. Zwischen dem 40. und 80. Tag nach der Lebertransplantation normalisierten sich die Werte für den Gesamtbilirubingehalt, lagen die Aktivitäten für die Transaminasen vergleichsweise niedrig und zeigten - mit Ausnahme der alkalischen Phosphatase - die Aktivitäten der Lactat-Dehydrogenase, Glutamat-Dehydrogenase und Cholinesterase einen annähernd konstanten Verlauf. Während dieses Zeitraums befand sich der Patient in einem relativ guten Allgemeinzustand, der seine Entlassung aus der stationären Behandlung rechtfertigte.

5. Nach der Entlassung kam es zu einer erneuten und stetigen Zunahme des Gesamtbilirubin-Gehaltes, der Aktivitäten der Transaminasen und der alkalischen Phosphatase. 
6. Nach der Wiederaufnahme deutete sich die zunehmende Verschlechterung der Leberfunktion durch einen stetigen Abfall der Cholinesterase an, während die Aktivitäten der übrigen Serumenzyme zum Teil erheblich zunahmen (alkalische Phosphatase, Transaminasen und Lactat-Dehydrogenase), sich im übrigen aber uneinheitlich verhielten.

7. Das Versagen der Funktion der transplantierten Leber infolge des Parenchymausfalls wurde an dem Absinken der Aktivitäten der Transaminasen und der alkalischen Phosphatase auf „Normalwerte“ sowie dem kurzfristigen und hohen Ansteigen der mitochondrialen Glutamat-Dehydrogenase und des Gesamtbilirubins erkennbar.

8. Diese Veränderungen waren von komatösen $\mathrm{Zu}$ ständen begleitet, die nach wenigen Tagen zum Exitus führten.

\section{Verlaufsbeobacbtungen beim Patienten H.O.}

Das Verhalten des Gesamtbilirubins, des konjugierten Bilirubins, der alkalischen Phosphatase und der Transaminasen ist in Abbildung 4 dargestellt. Im Gegensatz zu dem Patienten V. G. war es bei dem Patienten H. O. möglich, die klinisch-chemischen Untersuchungen be-
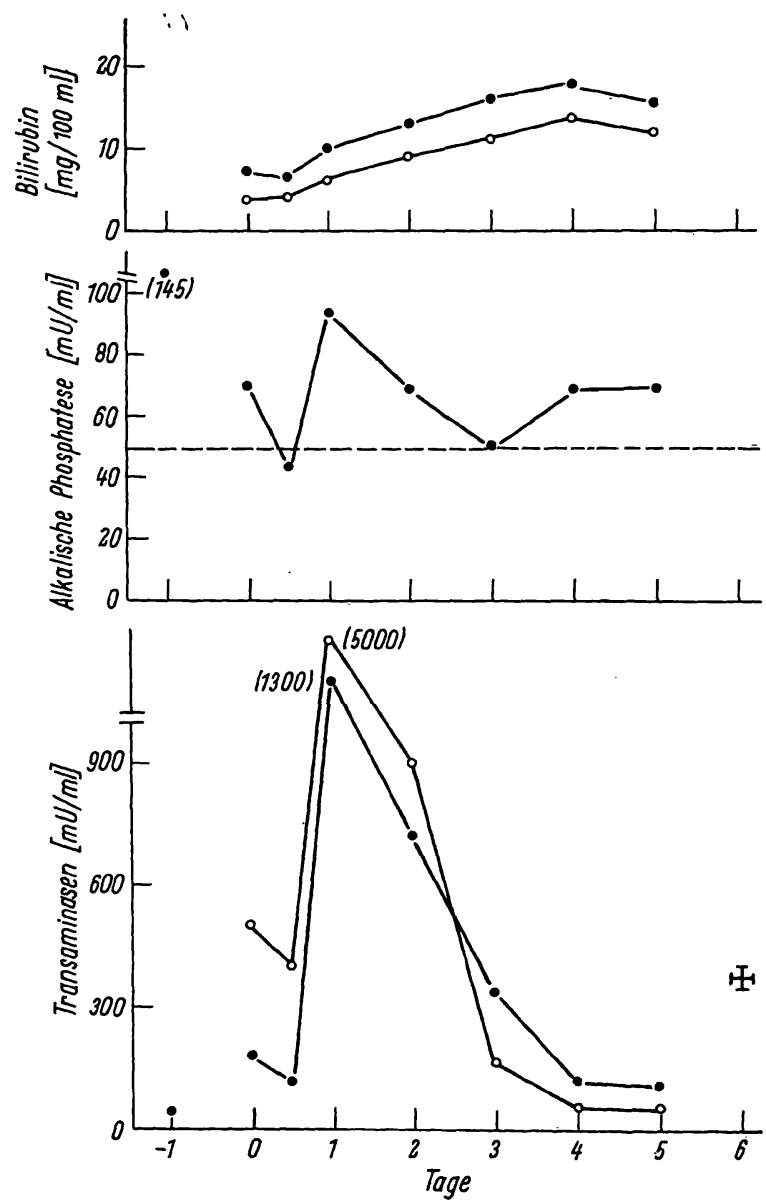

Abb. 4

Verhalten des Gesamtbilirubins $(\cdot-\circ)$ und des konjugierten $\left(0_{-}^{-}\right)$ Bilirubins (oben), der alkalischen Phosphatase (Mitte), der Alanin$(\bullet-\circ)$ und der Aspartat- ( $\circ)$ Aminotransferase (unten) im Serum eines 50jährigen Patienten ( $H . O$.) nach homologer Lebertransplantation. Die obere Begrenzung des Normalbereichs der alkalischen Phosphatase ist durch eine gestrichelte Linie gekennizeichnet. Am Tage 0 wurde die Transplantation durchgeführt
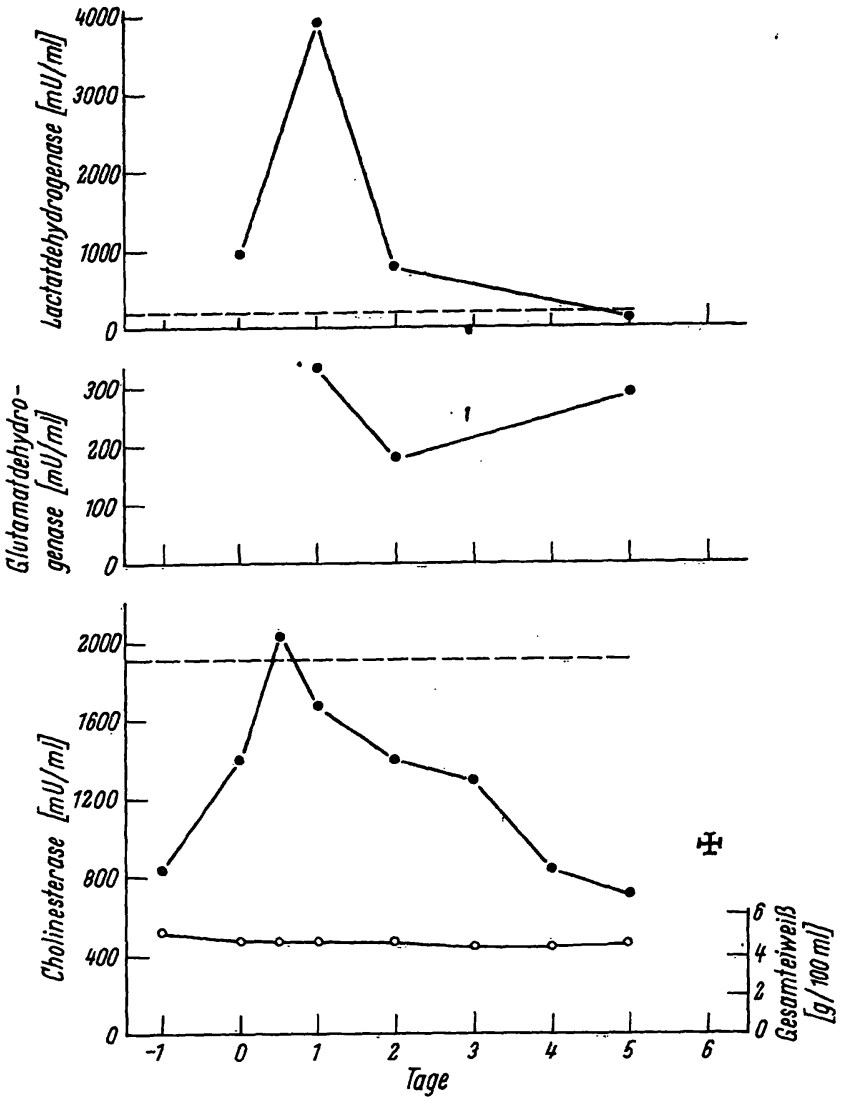

Abb. 5

Verhalten der Lactat-Dehydrogenase (oben), der Glutamat-Dehydrogenase (Mitte) und der Cholinesterase (unten) im Serum eines 50jährigen Patienten (H. O.) nach homologer Lebertransplantation. Die obere Begrenzung für den Normalbereich der Lactat-Dehydrogenase und die untere Begrenzung für den Normalbereich der Cholinesterase sind durch gestrichelte Linien gekennzeichnet. Am Täge 0 wurde die Transplantation durchgeführt

reits unmittelbar nach Beendigung der Einpflanzung des Spenderorgans durchzuführen. Bereits am 1. postoperativen Tag nahmen die Aktivitäten der AlaninAminotransferase auf $1300 \mathrm{mU} / \mathrm{ml}$ und der AspartatAminotransferase auf $5000 \mathrm{mU} / \mathrm{ml} \mathrm{zu}$. Dieser ungewöhnlich hohe Anstieg war, wie aus Abbildung 5 hervorgeht, von ebenfalls sehr hohen Aktivitäten der Lactat-Dehydrogenase und der Glutamat-Dehydrogenase begleitet. In den folgenden vier Tagen blieben die Aktivitäten der Glutamat-Dehydrogenase und der alkalischen Phosphatase weiterhin erhöht, während die Aktivitäten der Transaminasen, der Lactat-Dehydrogenase und der Cholinesterase abnahmen; gleichzeitig stieg der Bilirubingehalt stetig an. Tabelle 1 zeigt das Ver-

Tab. 1

Verhalten von Kalium, Natrium, Kreatinin und Harnstoff-Stickstoff im Serum eines 50 jährigen Patienten (H. O.) nach homologer Lebertm Serum eines Die in Klammern angegebenen Zahlen geben die niedrigsten und höchsten Werte an, die während der ersten 5 Stdn. nach Transhochsten Werte an, dion gemessen wurden

\begin{tabular}{ccccc}
\hline Tag & $\begin{array}{c}\text { Kalium } \\
{[\mathrm{m} \cdot \mathrm{Val} / l]}\end{array}$ & $\begin{array}{c}\text { Natrium } \\
{[\mathrm{mVal} / \mathrm{l}]}\end{array}$ & $\begin{array}{c}\text { Kreatinin } \\
{[\mathrm{mg} / 100 \mathrm{ml}]}\end{array}$ & $\begin{array}{c}\text { Harnstoff-N } \\
{[\mathrm{mg} / 100 \mathrm{~m} l]}\end{array}$ \\
\hline-1 & $\overline{6,1}$ & $\overline{132}$ & 3,0 & 112 \\
0 & $(3,8,-7,1)$ & $(122-140)$ & 6,3 & 75 \\
1 & 5,0 & 141 & 3,1 & 68 \\
2 & 6,4 & 150 & 3,7 & 58 \\
3 & 6,5 & 137 & 4,2 & 65 \\
4 & 6,4 & 147 & 4,4 & 90 \\
5 & 6,8 & 146 & 4,2 & 92 \\
\hline
\end{tabular}




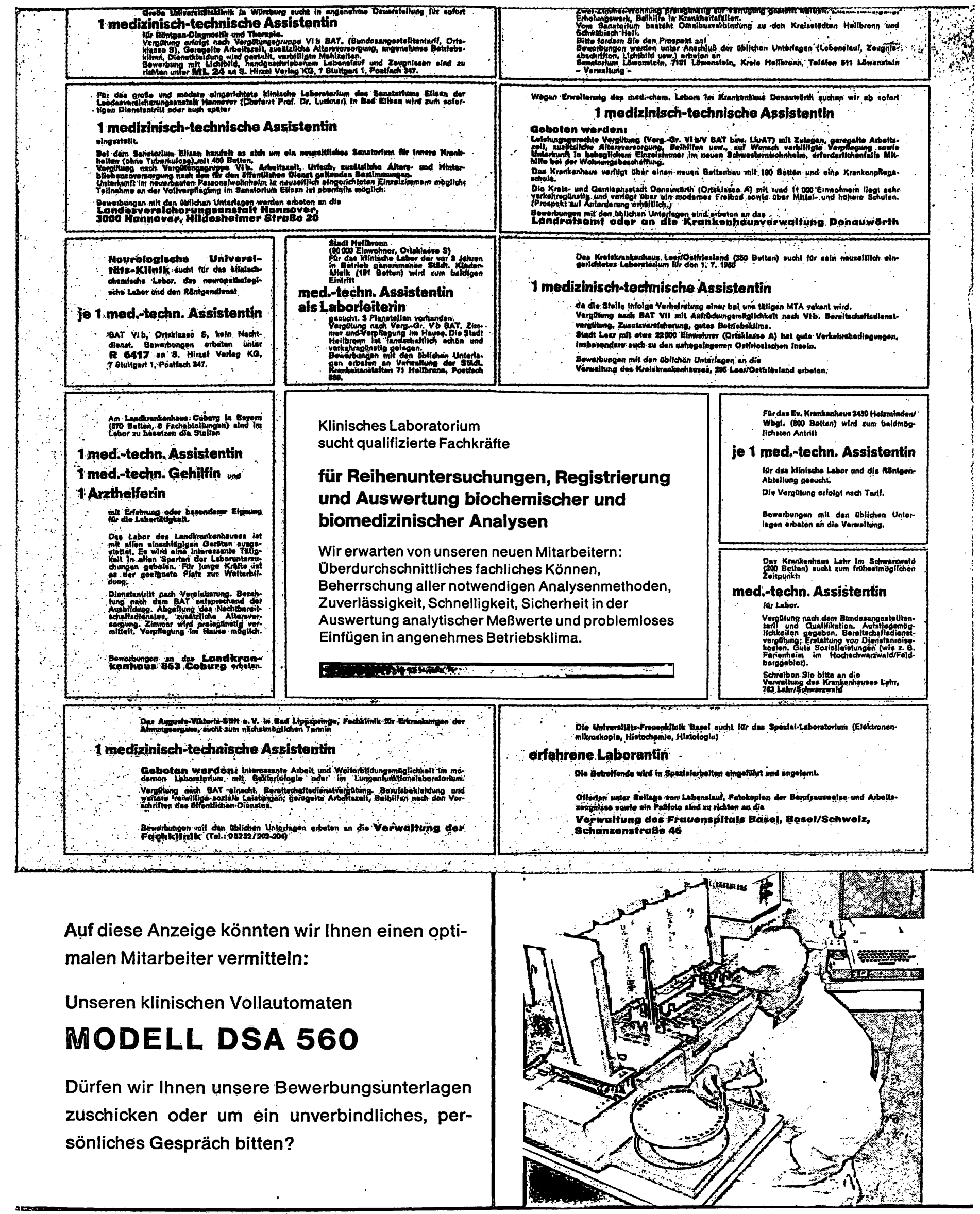
BECKMAN INSTRUMENTS GMBH 8 München 45, Frankfurter Ring 115, Tel.38871, Telex05-23823

Technische Büros: Berlin, Düsseldorf, Frankfurt, Hamburg, Hannover, München, Stuttgart

Internationale Niederlassungen: Fullerton/USA, Genf, Paris,

Glenrothes/Schottland, Tokio, Kapstadt, Wien, Amsterdam, Stockholm: 


\section{CECIL-FOTOMETER SYSTEME CE 303 und 404}

(CE 404 Filterfotometer) Die Fotometer sind auch als Einzelgeräte erhältlich. (CE 303 Gitterfotometer)

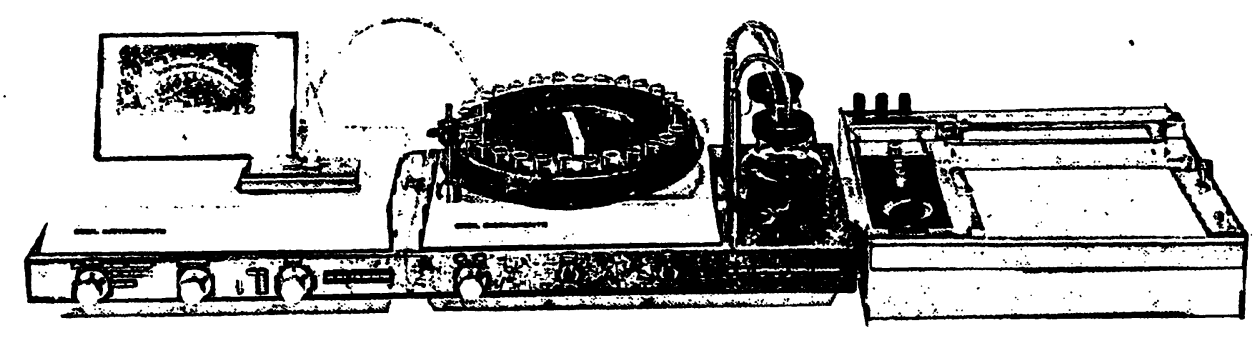

Bitte fordern Sie ausführliche Unterlagen an. Wir stellen Ihnen gerne ein Gerät zur Erprobung zur Verfügung.
Anwendungsgebiete:

Ḱlinische Chemie

Biochemie

Qualitätskontrolle

Bodenuntersuchungen

Lebensmittelchemie

Metallurgie

Wasserchemie

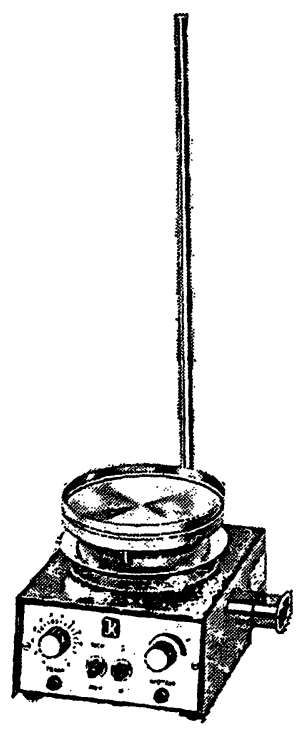

IKA-Combimag ${ }^{\circledR}$,

Magnetrührer im Baukastensystem aneinanderzureihen, magnetstark, preisgünstig, RCO, ohne Heizung, RCH (und RCHR), mit stufenloser Heizung, 2 Heizbereiche, $25-500 \mathrm{~W}$., auch für Steuerung durch Kontaktthermometer.

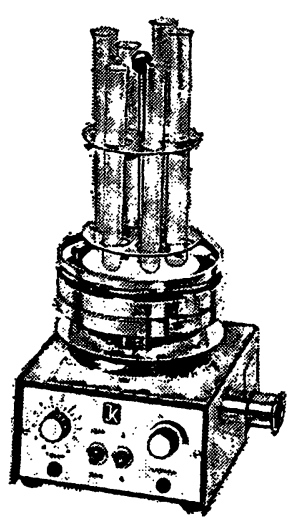

IKA-Schüttelaufsatz RCS,

ein ideales Zusatzgerät für IKA-Combimag-Magnetrührer zum intensiven Schütteln kleiner Mengen bis $1 \mathrm{~kg}$. Durch geeignete Halterungen können Reagenzgläser, Flaschen, Kolben und kleine Siebsätze geschüttelt werden.

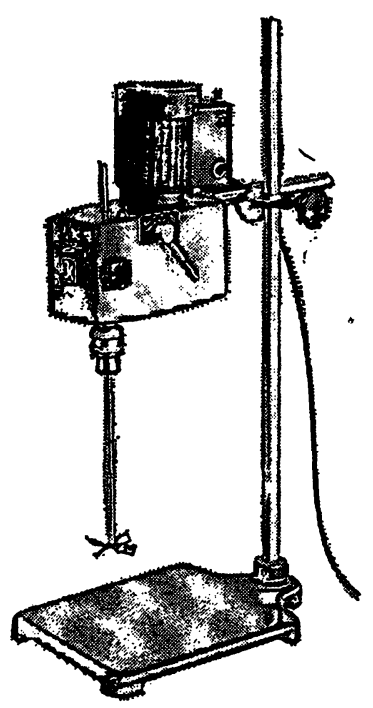

IKA-Rührmotor RM 18,

sehr leistungsfähiger, robuster Rührmotor mit Wechselstrommotor 25 Watt, mechanischer Drehzahlregelung von 25-2000 U.p.M., Drehzahl ablesbar. Mit Oberlastungsschutz. Biegsame Welle. 
halten von Kalium, Natrium, Kreatinin und Harnstoff; die Werte liegen - mit Ausnahme von Natrium - oberhalb der Norm, lassen jedoch während der postoperativen Phase keine Veränderungen erkennen. Die erhöhten Werte für Kalium sowie für Kreatinin und HarnstoffStickstoff sind Ausdruck eines bereits seit längerer Zeit bestehendenen Nierenschadens. Der Patient H. O. verstarb am 6. postoperativen Tag an einem Herzversagen. Nach Obduktion ergab die histologische Untersuchung der transplantierten Leber (Pathologisches Institut der Universität Bonn; Direktor Professor Dr. P. GEDIGK) Hinweise für eine beginnende Abstoßungsreaktion, jedoch keine Anhaltspunkte für einen Parenchymschaden. Dieser Befund steht im Einklang mit den klinisch-chemischen Untersuchungen und spricht gegen ein akutes Leberversagen.

\section{Vergleich der Verlaufsbeobachtungen}

Sowohl die Transaminasen als auch der Bilirubingehalt verhielten sich unmittelbar nach der Lebertransplantation bei beiden Patienten etwa gleich; ähnliches gilt für die Aktivitäten der alkalischen Phosphatase und Cholinesterase, während für die Lactat- und GlutamatDehydrogenase keine Aussagen gemacht werden können. Aus den Ergebnissen der klinisch-chemischen Befunde kann geschlossen werden, daß die erste akute Abstoßungsreaktion bei beiden Patienten etwa zum gleichen Zeitpunkt nach der Lebertransplantation begonnen haben dürfte; diese Annahme wird durch die histologischen Befunde der transplantierten Leber beim Patienten (H. O.) gestützt.

Von Interesse ist die Feststellung, daß die Aktivitäten der Transaminasen und der Glutamat-Dehydrogenase im
Serum des Patienten H. O. deutlich höher waren als beim Patienten V. G. So betrugen am 2. postoperativen Tage bei dem Patienten $\mathrm{H}$. O. die Aktivitäten der Alanin-Aminotransferase $720 \mathrm{mU} / \mathrm{ml}$ und der Aspartat-Aminotransferase $900 \mathrm{mU} / \mathrm{m} /$, während die entsprechenden Werte bei dem Patienten V. G. $450 \mathrm{mU} / \mathrm{m} l$ und $510 \mathrm{mU} / \mathrm{m} l$ waren.

Am 5. postoperativen Tage wurde für die GlutamatDehydrogenase bei dem Patienten $\mathrm{H}$. O. ein Wert von $344 \mathrm{mU} / \mathrm{ml}$ und bei dem Patienten V. G. ein Wert von $30 \mathrm{mU} / \mathrm{ml}$ ermittelt.

Ein ähnliches Verhalten der Transaminasen und der alkalischen Phosphatase wie bei den Patienten V. G. und $\mathrm{H}$. O. wurde auch von CALNE und Mitarbeitern (3) und von STarzL und Mitarbeitern (4) beobachtet. Die bisher ermittelten Werte für Bilirubin liegen - verglichen mit den Angaben in der Literatur $(3,4)-$ relativ hoch; eine Ausnahme machen die Bilirubinwerte des Patienten V. G. in der Zeit vom 50. bis 90. Tage nach der Lebertransplantation. Werte über die Aktivitäten der Cholinesterase, Glutamat- und LactatDehydrogenase nach homologer Lebertransplantation fehlen bisher in der Literatur. Wie besonders an der 206 tägigen Verlaufsbeobachtung bei dem Patienten V. G. gezeigt werden konnte, lassen sich aus dem Verhalten dieser drei Enzyme wichtige Schlußfolgerungen auf die Funktion der transplantierten Leber ziehen. Die hier dargelegten Befunde unterstreichen die Wichtigkeit klinisch-chemischer Verlaufbeobachtungen bei homologen Lebertransplantationen; dabei sollte neben den Transaminasen, der alkalischen Phosphatase der Lactat-Dehydrogenase und dem Bilirubingehalt besonders dem Verhalten der Glutamat-Dehydrogenase und der Cholinesterase Beachtung geschenkt werden.

\section{Literatur}

1. Gütgemann, A., K. H. Schriefers, G. Esser, T. S. Lee, K. J. Paquer und C. Käufer, Dtsch. med. Wschr. 94, 1713 (1969) - GürgemanN und Mitarbeiter in Vorbereitung. - 2. GürgemanN A., T. S. Lie, J. Breuer und H. Bréuer, diese Z. 7, 563 (1969). 3. Calne, R. Y., R. Williams, J. L. Dawson, I. D. Anseli, D. B. Evans, P. T. Flute, P. M. Herberison, V. Joysey, G. H. W.
Keates, R. P. Knill-Jones, S. A. Mason, P. R. Millard, J. R. Pena, B. D. Pentlow, J. R. Salaman, R. A. Sells und P. A. Cullum, Brit. Med. J. 541 (1968). - 4. Starzl, T. F., K. A. Porter, L. Brettschneider, I. Penn, P. Bell, C. W. Putnam und R. L. McGurre, Surg. Gyn. Obstetr. 128, 327 (1969).

Prof. Dr. H. Breuer Inst. f. Klin. Biochemie 5300 Bonn-Venusberg 